\title{
On Asymmetric Unification and the Combination Problem in Disjoint Theories
}

\author{
Serdar Erbatur ${ }^{1}$, Deepak Kapur ${ }^{2, \star}$, Andrew M. Marshall ${ }^{3}$, \\ Catherine Meadows ${ }^{4}$, Paliath Narendran ${ }^{5, \star \star}$, and Christophe Ringeissen ${ }^{6}$ \\ 1 Università degli Studi di Verona, Italy \\ 2 University of New Mexico, Albuquerque, NM, USA \\ 3 ASEE, Washington, DC, USA \\ 4 Naval Research Laboratory, Washington, DC, USA \\ ${ }^{5}$ University at Albany-SUNY, Albany, NY, USA \\ ${ }^{6}$ LORIA - INRIA Nancy-Grand Est, Nancy, France
}

\begin{abstract}
Asymmetric unification is a new paradigm for unification modulo theories that introduces irreducibility constraints on one side of a unification problem. It has important applications in symbolic cryptographic protocol analysis, for which it is often necessary to put irreducibility constraints on portions of a state. However many facets of asymmetric unification that are of particular interest, including its behavior under combinations of disjoint theories, remain poorly understood. In this paper we give a new formulation of the method for unification in the combination of disjoint equational theories developed by Baader and Schulz that both gives additional insights into the disjoint combination problem in general, and furthermore allows us to extend the method to asymmetric unification, giving the first unification method for asymmetric unification in the combination of disjoint theories.
\end{abstract}

\section{Introduction}

We examine the disjoint combination problem in the newly developed paradigm of asymmetric unification. This new unification problem was developed based on newly identified requirements arising from symbolic cryptographic protocol analysis [8. Its application involves unification-based exploration of a space in which the states obey rich equational theories that can be expressed as a decomposition $R \uplus E$, where $R$ is a set of rewrite rules that is confluent, terminating and coherent modulo $E$. However, in order to apply state space reduction techniques, it is usually necessary for at least part of this state to be in normal form, and to remain in normal form even after unification is performed. This requirement can be expressed as an asymmetric unification problem $\left\{s_{1}=\downarrow t_{1}, \ldots, s_{n}=\downarrow t_{n}\right\}$ where the $=\downarrow$ denotes a unification problem with the restriction that any unifier leaves the right-hand side of each equation irreducible (see Definition 4).

\footnotetext{
* Partially supported by the NSF grant CNS-0905222.

** Partially supported by the NSF grant CNS-0905286.
} 
The concept of asymmetric unification has its genesis in the unification method that is commonly used in symbolic analysis of cryptographic protocols. Here, two different requirements must be satisfied. The first is to have a generic unification algorithm that can be applied to a large class of equational theories that are encountered in cryptographic protocol analysis. The second is to guarantee that certain terms always be in normal form with respect to $R$ (see Section 1.1), so that it is possible to apply state space reduction techniques. This is done by decomposing the theory into $R \uplus E$ so that $R$ has the finite variant property [6] with respect to $E$, i.e., for any term $t$ there is a finite set of irreducible variants $V(t)$ of pairs $(u, \sigma)$, where $u$ is a term and $\sigma$ is a substitution, so that for each $(u, \sigma) \in V(t)$ we have $t \sigma \downarrow={ }_{E} u$ and for any substitution $\tau$ there is a $(u, \sigma) \in V(t)$ and a substitution $\rho$ such that $t \tau \downarrow={ }_{E} u \rho$. In other words, the set of variants gives a complete representation of the irreducible forms of $t$ under any substitution. A unification problem is then solved by computing the variants of each side and unifying those modulo $E$. This approach to unification is used in a number of tools, including ProVerif [4, OFMC [16], Maude-NPA [10], and Tamarin 15. More recently, it has been formalized in a procedure known as folding variant narrowing [12, which terminates if and only if the terms being unified have a finite number of variants.

Although variant narrowing is sound and complete for theories with the finite variant property, it is not optimally efficient. In [7] it is pointed out that the issue can often be addressed by computing the set of variants of only one side of a unification problem $s={ }^{?} t$, replacing it with a new asymmetric problem $s={ }^{\downarrow} t_{1}, \ldots, s={ }^{\downarrow} t_{n}$. One may then apply more efficient special-purpose asymmetric unification algorithms that satisfy the irreducibility constraints. Recent work on asymmetric algorithms for exclusive-or [14, [8] and free Abelian groups [14 indicate that such algorithms can lead to significant enhancement of performance.

Although asymmetric unification has the potential of playing an important role in cryptographic protocol analysis, and possibly other unification-based state exploration as well, it is still not that well understood. Until the development of special-purpose algorithms for exclusive-or and free Abelian group theories mentioned above, the only known asymmetric unification algorithm was variant narrowing. Since then, some better understanding has been developed. For Example, we know that asymmetric unification is strictly harder than "symmetric" unification. In particular, there are theories for which symmetric unification is decidable and asymmetric unification is not. Still, there are many questions that remain to be answered. One of the most important of these unanswered questions is the problem of asymmetric unification in a combination of theories, in particular how to produce an algorithm for the combined theory by combining algorithms for the separate theories. This is particularly significant for cryptographic protocol analysis. Cryptographic protocols generally make use of more than one cryptoalgorithm. Often, these cryptoalgorithms can be described in terms of disjoint equational theories. In the case in which the algorithm used is variant narrowing, the problem is straightforward. If the combination of two 
theories with the finite variant property also has the finite variant property, then one applies variant narrowing. However, in attempting to combine theories with special-purpose algorithms, the path is less clear. This is an important point with respect to efficiency since, as pointed out above, special-purpose asymmetric algorithms have the promise of being more efficient than variant narrowing.

In this paper we take the first step to solving this problem, by showing that the combination method for the unification problem in disjoint equational theories developed by Baader and Schulz in [2] can be modified and extended to the asymmetric unification paradigm, thus providing the first general combination method for this new paradigm. The only restrictions on this new method are those inherited from the asymmetric unification problem and those inherited from Baader and Schulz. From [2] we require that the algorithms being combined solve the asymmetric unification with linear constant restriction problem, although we show this reduces to solving the general asymmetric unification problem.

Do to space restrictions some proof details have been omitted. Please see the technical report version for full proofs [9].

\subsection{Preliminaries}

We use the standard notation of equational unification [3] and term rewriting systems [1]. The set of $\Sigma$-terms, denoted by $T(\Sigma, \mathcal{X})$, is built over the signature $\Sigma$ and the (countably infinite) set of variables $\mathcal{X}$. The terms $\left.t\right|_{p}$ and $t[u]_{p}$ denote respectively the subterm of $t$ at the position $p$, and the term $t$ having $u$ as subterm at position $p$. The symbol of $t$ occurring at the position $p$ (resp. the top symbol of $t$ ) is written $t(p)$ (resp. $t(\epsilon)$ ). The set of positions of a term $t$ is denoted by $\operatorname{Pos}(t)$, the set of non variable positions for a term $t$ over a signature $\Sigma$ is denoted by $\operatorname{Pos}(t)_{\Sigma}$. A $\Sigma$-rooted term is a term whose top symbol is in $\Sigma$. The set of variables of a term $t$ is denoted by $\operatorname{Var}(t)$. A term is ground if it contains no variables. A term $t$ is linear if each variable of $t$ occurs only once in $t$.

A $\Sigma$-substitution $\sigma$ is an endomorphism of $T(\Sigma, \mathcal{X})$ denoted by $\left\{x_{1} \mapsto t_{1}, \ldots\right.$, $\left.x_{n} \mapsto t_{n}\right\}$ if there are only finitely many variables $x_{1}, \ldots, x_{n}$ not mapped to themselves. We call the domain of $\sigma$ the set of variables $\left\{x_{1}, \ldots, x_{n}\right\}$ and the range of $\sigma$ the set of terms $\left\{t_{1}, \ldots, t_{n}\right\}$. Application of a substitution $\sigma$ to a term $t$ (resp. a substitution $\phi$ ) may be written $t \sigma$ (resp. $\phi \sigma)$.

Given a first-order signature $\Sigma$, and a set $E$ of $\Sigma$-axioms (i.e., pairs of $\Sigma$ terms, denoted by $l=r$ ), the equational theory $={ }_{E}$ is the congruence closure of $E$ under the law of substitutivity. By a slight abuse of terminology, $E$ will be often called an equational theory. An axiom $l=r$ is variable-preserving if $\operatorname{Var}(l)=\operatorname{Var}(r)$. An axiom $l=r$ is linear (resp. collapse-free) if $l$ and $r$ are linear (resp. non-variable terms). An equational theory is variable-preserving (resp. linear/ collapse-free) if all its axioms are variable-preserving (resp. linear/ collapse-free). An equational theory $E$ is finite if for each term $t$, there are finitely many terms $s$ such that $t={ }_{E} s$. 
A $\Sigma$-equation is a pair of $\Sigma$-terms denoted by $s=? t$. An $E$-unification problem is a set of $\Sigma$-equations, $\mathcal{S}=\left\{s_{1}={ }^{?} t_{1}, \ldots, s_{m}={ }^{?} t_{m}\right\}$. The set of variables of $\mathcal{S}$ is denoted by $\operatorname{Var}(\mathcal{S})$.

A solution to $\mathcal{S}$, called an E-unifier, is a substitution $\sigma$ such that $s_{i} \sigma={ }_{E} t_{i} \sigma$ for all $1 \leq i \leq m$. A substitution $\sigma$ is more general modulo $E$ than $\theta$ on a set of variables $V$, denoted as $\sigma \leq_{E}^{V} \theta$, if there is a substitution $\tau$ such that $x \sigma \tau={ }_{E} x \theta$ for all $x \in V$. Two substitutions $\theta_{1}$ and $\theta_{2}$ are equivalent modulo $E$ on a set of variables $V$, denoted as $\theta_{1}={ }_{E}^{V} \theta_{2}$, if and only if $x \theta_{1}={ }_{E} x \theta_{2}$ for all $x \in V$. A complete set of E-unifiers of $\mathcal{S}$ is a set of substitutions denoted by $\operatorname{CSU}_{E}(\mathcal{S})$ such that each $\sigma \in C S U_{E}(\mathcal{S})$ is an $E$-unifier of $\mathcal{S}$, and for each $E$-unifier $\theta$ of $\mathcal{S}$, there exists $\sigma \in C S U_{E}(\mathcal{S})$ such that $\sigma \leq_{E}^{\operatorname{Var}(\mathcal{S})} \theta$.

Equational unification problems are classified based on the function symbols that appear in them, i.e., their signature $(S i g)$. An $E$-unification problem $S$ is elementary if and only if $\operatorname{Sig}(S)=\operatorname{Sig}(E) . S$ is called an $E$-unification problem with constants if $\operatorname{Sig}(S) \backslash \operatorname{Sig}(E)$ contains only free constants. Finally, if there are uninterpreted function symbols in $\operatorname{Sig}(S) \backslash \operatorname{Sig}(E), S$ is called a general $E$ unification problem.

Let $E_{1}$ and $E_{2}$ be two equational theories built over the disjoint signatures $\Sigma_{1}$ and $\Sigma_{2}$. The elements of $\Sigma_{i}$ will be called $i$-symbols. A term $t$ is an $i$-term if and only if it is of the form $t=f\left(t_{1}, \ldots, t_{n}\right)$ for an $i$-symbol $f$ or $t$ is a variable. An $i$-term is pure (or an $i$-pure term) if it only contains $i$-symbols and variables. An equation $s={ }^{?} t$ is $i$-pure (or just pure) iff there exists an $i$ such that $s$ and $t$ are $i$-pure terms or variables. A subterm $s$ of an $i$-term $t$ is called an alien subterm (or just alien) of $t$ iff it is a non-variable $j$-term, $j \neq i$, such that every proper superterm of $s$ in $t$ is an $i$-term. A unification problem $S$ is an $i$-pure problem if all equations in $S$ are $i$-pure.

Definition 1. Let $\Gamma$ be an E-unification problem, let $\mathcal{X}$ denote the set of variables occurring in $\Gamma$ and $\mathcal{C}$ the set of free constants occurring in $\Gamma$. For a given linear ordering $<$ on $\mathcal{X} \cup \mathcal{C}$, and for each $c \in \mathcal{C}$ define the set $V_{c}$ as $\{x \mid x$ is a variable with $x<c\}$. An $E$-unification problem with linear constant restriction $(L C R)$ is an E-unification problem with constants, $\Gamma$, where each constant $c$ in $\Gamma$ is equipped with a set $V_{c}$ of variables. A solution of the problem is an E-unifier $\sigma$ of $\Gamma$ such that for all $c, x$ with $x \in V_{c}$, the constant $c$ does not occur in $x \sigma$. We call $\sigma$ an $E$-unifier with linear constant restriction.

A rewrite rule is an ordered pair $l \rightarrow r$ such that $l, r \in T(\Sigma, \mathcal{X})$ and $l \notin \mathcal{X}$. We use $R$ to denote a term rewrite system which is defined as a set of rewrite rules. The rewrite relation on $T(\Sigma, \mathcal{X})$, written $t \rightarrow_{R} s$, hold between $t$ and $s$ iff there exists a non-variable $p \in \operatorname{Pos}_{\Sigma}(t), l \rightarrow r \in R$ and a substitution $\sigma$, such that $\left.t\right|_{p}=l \sigma$ and $s=t[r \sigma]_{p}$. The relation $\rightarrow_{R / E}$ on $T(\Sigma, \mathcal{X})$ is $=_{E} \circ \rightarrow_{R} \circ=_{E}$. The relation $\rightarrow_{R, E}$ on $T(\Sigma, \mathcal{X})$ is defined as: $t \rightarrow_{R, E} t^{\prime}$ if there exists a position $p \in \operatorname{Pos}_{\Sigma}(t)$, a rule $l \rightarrow r \in R$ and a substitution $\sigma$ such that $\left.t\right|_{p}={ }_{E} l \sigma$ and $t^{\prime}=t[r \sigma]_{p}$. The transitive (resp. transitive and reflexive) closure of $\rightarrow_{R, E}$ is denoted by $\rightarrow_{R, E}^{+}$(resp. $\rightarrow_{R, E}^{*}$ ). A term $t$ is $\rightarrow_{R, E}$ irreducible (or in $R, E$ normal form) if there is no term $t^{\prime}$ such that $t \rightarrow_{R, E} t^{\prime}$. If $\rightarrow_{R, E}$ is confluent and terminating we denote the irreducible version of a term, $t$, by $t \rightarrow_{R, E}^{!}$or $t \downarrow_{R, E}$. 
Definition 2. A rewrite rule $l \rightarrow r$ is duplicating if $r$ contains more occurrences of some variable than $l$; otherwise, $l \rightarrow r$ is non-duplicating. We say that $R$ is non-duplicating if every $l \rightarrow r \in R$ is non-duplicating

Definition 3. We call $(\Sigma, E, R)$ a decomposition of an equational theory $\Delta$ over a signature $\Sigma$ if $\Delta=R \uplus E$ and $R$ and $E$ satisfy the following conditions:

1. $E$ is variable preserving, i.e., for each $s=t$ in $E$ we have $\operatorname{Var}(s)=\operatorname{Var}(t)$.

2. E has a finitary and complete unification algorithm. That is, an algorithm that produces a finite complete set of unifiers.

3. For each $l \rightarrow r \in R$ we have $\operatorname{Var}(r) \subseteq \operatorname{Var}(l)$.

4. $R$ is confluent and terminating modulo $E$, i.e., the relation $\rightarrow_{R / E}$ is confluent and terminating.

5. $\rightarrow_{R, E}$ is E-coherent, i.e., $\forall t_{1}, t_{2}, t_{3}$ if $t_{1} \rightarrow_{R, E} t_{2}$ and $t_{1}=_{E} t_{3}$ then $\exists t_{4}, t_{5}$ such that $t_{2} \rightarrow_{R, E}^{*} t_{4}, t_{3} \rightarrow_{R, E}^{+} t_{5}$, and $t_{4}={ }_{E} t_{5}$.

This definition is inherited directly from 8] where Asymmetric unification and the corresponding theory decomposition are first defined. The last restrictions ensure that $s \rightarrow{ }_{R / E}^{!} t$ iff $s \rightarrow !_{R, E} t$ (see [1], [8]).

Definition 4 (Asymmetric Unification). Given a decomposition $(\Sigma, E, R)$ of an equational theory, a substitution $\sigma$ is an asymmetric $R$, E-unifier of a set $\mathcal{S}$ of asymmetric equations $\left\{s_{1}={ }^{\downarrow} t_{1}, \ldots, s_{n}={ }^{\downarrow} t_{n}\right\}$ iff for each asymmetric equations $s_{i}={ }^{\downarrow} t_{i}, \sigma$ is an $(E \cup R)$-unifier of the equation $s_{i}={ }^{?} t_{i}$ and $\left(t_{i} \downarrow_{R, E}\right) \sigma$ is in $R, E$-normal form. A set of substitutions $\Omega$ is a complete set of asymmetric $R, E$-unifiers of $\mathcal{S}$ (denoted $C S A U_{R \cup E}(\mathcal{S})$ or just $C S A U(\mathcal{S})$ if the background theory is clear) iff: (i) every member of $\Omega$ is an asymmetric $R$, E-unifier of $\mathcal{S}$, and (ii) for every asymmetric $R, E$-unifier $\theta$ of $\mathcal{S}$ there exists a $\sigma \in \Omega$ such that $\sigma \leq_{E}^{\operatorname{Var}(\mathcal{S})} \theta$.

Example 1. Let $R=\{x \oplus 0 \rightarrow x, x \oplus x \rightarrow 0, x \oplus x \oplus y \rightarrow y\}$ and $E$ be the $A C$ theory for $\oplus$. Consider the equation $y \oplus x=^{\downarrow} x \oplus a$, the substitution $\sigma_{1}=\{y \mapsto a\}$ is an asymmetric solution but, $\sigma_{2}=\{x \mapsto 0, y \mapsto a\}$ is not.

Definition 5 (Asymmetric Unification with Linear Constant Restriction). Let $\mathcal{S}$ be a set of of asymmetric equations with some LCR. A substitution $\sigma$ is an asymmetric $R, E$-unifier of $\mathcal{S}$ with $L C R$ iff $\sigma$ is an asymmetric solution to $\mathcal{S}$ and $\sigma$ satisfies the $L C R$.

\section{Combining Asymmetric Unification Algorithms}

Here we modify and extend the method for unification in the union of disjoint equational theories, developed by Baader and Schulz 2, to the combination of asymmetric unification algorithms in the union of disjoint equational theories. 
Problem Description: Let $\Delta_{1}$ and $\Delta_{2}$ denote two equational theories with disjoint signatures $\Sigma_{1}$ and $\Sigma_{2}$. Let $\Delta$ be the combination, $\Delta=\Delta_{1} \cup \Delta_{2}$, of the two theories having signature $\Sigma_{1} \cup \Sigma_{2}$. Let $A_{i}, i \in\{1,2\}$, be an asymmetric $\Delta_{i}$-unification with linear constants restriction algorithm. We then give an algorithm which uses $A_{1}$ and $A_{2}$ to solve the elementary asymmetric unification problem over $\Delta$. Recall that elementary implies that terms can only contain symbols in the signature of the theory or variables. But this is not restrictive, if we wish to have additional free functional symbols, these function symbols define a new empty theory and lead to another combination. Therefore, in what follows we will assume that a problem, $\Gamma_{0}$, in the combined theory $\Delta$, is an elementary asymmetric $\Delta$-unification problem. In order to satisfy the requirements for asymmetric unification we make the following assumptions.

Restrictions: for each constituent theory $\left(\Sigma_{i}, \Delta_{i}\right)$ :

1. There is a decomposition $\Delta_{i}=R_{i} \uplus E_{i}$ and $u \rightarrow_{R_{i}, E_{i}}^{!} v$ iff $u \longrightarrow !_{R_{i} / E_{i}}^{!} v$ (see note (2) below).

2. $E_{i}$ is collapse-free and there exists a finitary $E_{i}$-unification algorithm.

3. There exists a finitary complete asymmetric $\Delta_{i}$-unification algorithm with linear constants restriction, $A_{i}$ (see note (3) below).

4. Variables are $\rightarrow_{R_{i}, E_{i}}$-normal forms.

5 . Each $R_{i}$ is non-duplicating.

Notes on the Restrictions:

1. All Restrictions, except (3), are due to the asymmetric unification definition.

2. The definition of decomposition requires that $\longrightarrow_{R_{i} / E_{i}}$ be confluent and terminating. Thus, if $u \rightarrow{\stackrel{R_{i}, E_{i}}{ }} v$ iff $u \longrightarrow !_{R_{i} / E_{i}}^{!} v$, we have that $\rightarrow_{R_{i}, E_{i}}$ is also confluent and terminating.

3 . We show in Section 2.5 that there exists an asymmetric $\Delta_{i}$-unification algorithm with linear constants restriction if there exists a general asymmetric $\Delta_{i}$-unification algorithm.

According to our Restrictions, $E_{1}$ and $E_{2}$ are both variable preserving and collapse-free. Consequently, we have the following property:

Lemma 1. $t \neq_{E_{1} \cup E_{2}}$ s, if $t$ is a non-variable $i$-term and $s$ is a non-variable $j$-term, $j \neq i$.

\subsection{Rewriting in the Combined Theory}

The definition of asymmetric unification in the combined theory $\Delta$, where $\Delta=$ $\Delta_{1} \cup \Delta_{2}$, requires us to not only find $\Delta$-unifiers but also decide if a term is in $\rightarrow\left(R_{1} \cup R_{2}\right),\left(E_{1} \cup E_{2}\right)$ normal form. Therefore, we need to first ensure the modularity of rewriting, i.e., ensure we can compute $\longrightarrow\left(R_{1} \cup R_{2}\right),\left(E_{1} \cup E_{2}\right)$-normal forms.

Consider now the combined theory $(\Sigma, \Delta)$, where $\Sigma=\Sigma_{1} \cup \Sigma_{2}$ and $\Delta=\Delta_{1} \cup$ $\Delta_{2}$. Let $\mathcal{R}=R_{1} \cup R_{2}$ and $\mathcal{E}=E_{1} \cup E_{2}$. Therefore, $\rightarrow_{\mathcal{R}, \mathcal{E}}$ denotes $\rightarrow_{R_{1} \cup R_{2}, E_{1} \cup E_{2}}$.

Theorem 1. $\rightarrow_{\mathcal{R}, \mathcal{E}}=\longrightarrow_{R_{1}, E_{1}} \cup \longrightarrow_{R_{2}, E_{2}}$ 
Proof. Follows from the the fact that $\Sigma_{1} \cap \Sigma_{2}=\emptyset$ and $\mathcal{E}=E_{1} \cup E_{2}$ is variable preserving and collapse-free.

The relation $\longrightarrow R_{i}, E_{i}$ is decidable for each sub-theory due to the assumption that $\longrightarrow R_{1}, E_{1}$ is convergent. Therefore we obtain the following corollary to Theorem 1 .

Corollary 1. The relation $\longrightarrow_{\mathcal{R}, \mathcal{E}}$ is decidable.

Note, with respect to termination, $R_{1} \cup R_{2}$ is non-duplicating, this is due to the disjoint theories and the fact that each $R_{i}$ is non-duplicating by assumption. Since $R_{1} \cup R_{2}$ is non-duplicating termination is obtained due to the results of [17], where it is shown that non-duplicating implies termination in the combination of terminating rewrite systems. Next we would like to know that $\longrightarrow \mathcal{R}, \mathcal{E}$ is complete with respect to $\longrightarrow \mathcal{R} / \mathcal{E}$, i.e., $u \rightarrow !_{R_{i}, E_{i}} v$ iff $u \longrightarrow !_{R_{i} / E_{i}} v$, which is not true in general. For this to be true we need to know that $\longrightarrow_{\mathcal{R}, \mathcal{E}}$ is $\mathcal{E}$-coherent, which implies the result (see [1] ).

Lemma 2. If there exist terms $t_{0}, t_{1}$ and $t_{2}$ such that $t_{0} \leftrightarrow_{\mathcal{E}}^{*} t_{2}$ and $t_{0} \rightarrow_{\mathcal{R}, \mathcal{E}} t_{1}$ then there exists a term $t_{3}$ such that $t_{2} \rightarrow_{\mathcal{R}, \mathcal{E}} t_{3}$.

Proof. This can be shown via an induction argument relying on the fact that $\rightarrow_{R_{i}, E_{i}}$ is coherent modulo $E_{i}$. See [9] for the full proof.

Theorem 2. $\rightarrow_{\mathcal{R}, \mathcal{E}}$ is $\mathcal{E}$-coherent.

Proof. If $t_{0} \rightarrow_{\mathcal{R}, \mathcal{E}} t_{1}$ and $t_{0}={ }_{\mathcal{E}} t_{2}$, then by Lemma 2, there exists a term, $t_{3}$, such that $t_{2} \rightarrow_{\mathcal{R}, \mathcal{E}} t_{3}$. Thus, $t_{1} \longleftrightarrow R_{1} \cup R_{2} \cup E_{1} \cup E_{2} t_{3}$. Now the combined system has the properties (normal form variables, $E_{i}$ collapse-free, and disjoint signatures) such that the Church-Rosser result in 13 applies. This implies the existence of terms $t_{4}$ and $t_{5}$ such that $t_{1} \rightarrow_{\mathcal{R}, \mathcal{E}}^{*} t_{4}, t_{3} \rightarrow_{\mathcal{R}, \mathcal{E}}^{*} t_{5}$ and $t_{4}=\mathcal{E} t_{5}$.

Therefore, based on Corollary 1 and Theorem 2, $u \rightarrow_{\mathcal{R}, \mathcal{E}}^{*} v$ iff $u \rightarrow_{\mathcal{R} / \mathcal{E}}^{*} v$ which implies the following:

Theorem 3. $t={ }_{\mathcal{R} \cup \mathcal{E}} s$ iff $t \downarrow_{\mathcal{R}, \mathcal{E}}={ }_{\mathcal{E}} s \downarrow_{\mathcal{R}, \mathcal{E}}$

\subsection{Asymmetry in the Projection of Terms}

Now that we have established the modular results for rewriting we can use the well defined normal forms to define projections onto pure terms. Later we will use the bijection defined below to prove that if the original problem has a solution then there exists solutions to the pure sub-problems. This is accomplished by mapping the combined solution into two pure solutions. In order for this to work we also need to ensure that equality modulo $E$ and asymmetric restrictions are maintained after the mapping is applied. Let $\mathcal{X}$ and $\mathcal{Y}$ be disjoint sets of variables that are countably infinite. Let $T(\Sigma, \mathcal{X})$ be the set of $\Sigma_{1} \cup \Sigma_{2}$-terms over $\mathcal{X}$. We define a bijection

$$
\pi:\left(T(\Sigma, \mathcal{X}) \downarrow_{\mathcal{R}, \mathcal{E}}\right)_{/=\mathcal{E}} \rightarrow \mathcal{Y}
$$


The bijection $\pi$ induces two mappings $\pi_{1}$ and $\pi_{2}$ of terms in $T(\Sigma, \mathcal{X})$ to terms in $T(\Sigma, \mathcal{Y})$ as follows. For each $x \in \mathcal{X}, x^{\pi_{1}}:=\pi(x)$. If $t=f\left(t_{1}, \ldots, t_{n}\right)$ for a 1-symbol $f$, then $t^{\pi_{1}}:=f\left(t_{1}^{\pi_{1}}, \ldots, t_{n}^{\pi_{1}}\right)$. If $t$ is a 2 -term then $t^{\pi_{1}}:=y$ where $y=\pi\left([s]_{\mathcal{E}}\right)$ for the unique $\rightarrow_{\mathcal{R}, \mathcal{E}}$-irreducible term $s$ of $t$, where $[s]_{\mathcal{E}}$ denotes the equivalence class of $s$ modulo $\mathcal{E}$. The mapping $\pi_{2}$ is defined analogously.

Given a substitution $\sigma, \sigma^{\pi_{i}}$ denotes the abstraction defined by $\sigma^{\pi_{i}}(x)=$ $(\sigma(x))^{\pi_{i}}$, for all $x$ is the domain of $\sigma$. These two mapping can be seen as projections from mixed terms into pure terms. More informally, we can view an $i$-abstraction as method for converting a mixed term into a pure term by replacing the alien subterms with variables. Recall that we assume that variables are $\longrightarrow R_{i}, E_{i}$-irreducible and thus by modularity $\rightarrow_{\mathcal{R}, \mathcal{E}}$-irreducible. As in $[2$ we can also define the inverse, $\pi^{-1}$ of $\pi$ as a substitution that maps the variables $y \in \mathcal{Y}$ back to terms $\pi^{-1}(y)$ and is the identity on all other variables. Note that, $\pi^{-1}\left(t^{\pi_{i}}\right)=_{\mathcal{E}} t$, if $t$ is in $\mathcal{R}, \mathcal{E}$-normal form or an $i$-term with normal form aliens.

Theorem 4. Let $s$ and $t$ be $i$-pure terms. Let $t$ and $\sigma$ be in $\mathcal{R}, \mathcal{E}$-normal form, such that $s \sigma={ }_{\Delta} t \sigma$. Then $s \sigma^{\pi_{i}}={ }_{\Delta_{i}} t \sigma^{\pi_{i}}$ and $t \sigma$ is in $\mathcal{R}, \mathcal{E}$-normal form iff $t \sigma^{\pi_{i}}$ is in $R_{i}, E_{i}$-normal form.

Proof. This result follows from the disjoint signatures and the fact that $\mathcal{E}$ is variable preserving. See [9] for the full proof details.

\subsection{Asymmetric Unification with Linear Constant Restriction}

We present the combination Algorithm, AsymComb, in Figure 1. Let us first give a rough, intuitive overview of the steps. First, equations are purified using variable abstraction and splitting (steps 1 and 2 ). This ensures that the original problem is separated into pure problems which can be solved by the algorithms for the pure theories. Next, a variable identification is non-deterministically chosen, allowing for the testing all the ways the variables may be equated to other variables. Then, a linear ordering and theory indices are non-deterministically chosen. Note that a shared variable can only "belong" exclusively to one theory. Since we don't know beforehand what variable belongs to which theory the non-deterministic selections allow us to check all the possibilities. In addition, each solution to the original problem will correspond to one or more linear ordering among the variables. Next, the problem is split into two pure problems where the linear ordering defines a linear constant restriction. The pure problems are solved by asymmetric unification algorithms with linear constant restriction. The solutions returned by the sub-algorithms are combined into solutions for the original problem. The Algorithm AsymComb (cf. Figure1) must also ensure that we only combine a specific type of unifier, which ensures asymmetry.

The notions of identification, theory indexes and linear constant restrictions, have all been used in [2] (see Section 1.1] for definitions). In order to handle the asymmetry restriction we introduce two additional notions, which ensure pure problem solutions having these properties will result in asymmetric combined solutions. 
Input: $\Gamma_{0}$, the initial unification problem over the signature $\Sigma_{1} \cup \Sigma_{2}$, where we assume the right hand sides of the equations are normalized.

1. Variable Abstraction: Let $s=^{\downarrow} t \in \Gamma_{0}$.

(a) Right Abstraction: For each alien subterm $t_{1}$ of $t$, let $x$ be a variable not occurring in the current system and let $t^{\prime}$ be the term obtained from replacing $t_{1}$ by $x$ in $t$. Then the original equation is replaced by two equations $s=t^{\prime}$ and $x={ }^{\downarrow} t_{1}$.

(b) Left Abstraction: For each alien subterm $s_{1}$ of $s$ let $x$ be a variable not occurring in the current system and let $s^{\prime}$ be the term obtained from replacing $s_{1}$ by $x$ in $s$. Then the original equation is replaced by two equations $s^{\prime}=^{\downarrow} t$ and $s_{1}={ }^{\downarrow} x$.

The output is a system $\Gamma_{1}$ such that all terms are pure.

2. Split non-pure equations: Each non-pure equation of the form $s=^{\downarrow} t$ is replaced by two equations $s=^{\downarrow} x, x=^{\downarrow} t$ where $x$ is always a new variable. The results is a system $\Gamma_{2}$ of pure equations.

3. Variable Identification: Consider all the possible partitions of the set of variables. Each partition produces a new system $\Gamma_{3}$ as follows. The variables in each class of the partition are "identified" with each other by choosing an element of the class as a representative and replacing in the system all occurrences of variables in each class by their representative.

4. Choose an ordering and Theory index: For each $\Gamma_{3}$ we consider all the possible strict orderings $<$ on the variables of the system and all mappings $i$ ind from the set of variables into the set of indices $\{1,2\}$. Each pair $(<$, ind $)$ yields a new system $\Gamma_{4}$.

5. Split the system: Each $\Gamma_{4}$ is split into two systems $\Gamma_{5,1}$ and $\Gamma_{5,2}$, the first containing only 1 -equations and the second only 2 -equations. In the system $\Gamma_{5, i}$ the variables of index $j \neq i$ are treated as constants. Each $\Gamma_{5, i}$ is now a unification problems with linear constant restriction, where the linear ordering $<$ defines the set $V_{c}$ for each constant $c$ corresponding to an index $j \neq i$ variable.

6. Compute $M_{i, j}$ : For the initial system $\Gamma_{0}$ let $\left\{\left(\Gamma_{5,1}^{1}, \Gamma_{5,2}^{1}\right), \ldots,\left(\Gamma_{5,1}^{n}, \Gamma_{5,2}^{n}\right)\right\}$ be the output of the decomposition. For $i=1, \ldots, n$ and $j=1,2$, let $M_{i, j}=$ $C S A U_{\Delta_{j}}\left(\Gamma_{5, j}^{i}\right)$ produced by Algorithm $A_{j}$, where substitutions that are noninjective and not theory-preserving are discarded.

7. Output: For $i=1, \ldots, n$ the set of substitutions $\sigma_{1} \odot \sigma_{2}$ such that $\sigma_{1} \in M_{i, 1}$ and $\sigma_{2} \in M_{i, 2}$.

Fig. 1. Algorithm AsymComb

Definition 6. (Injective)

A substitution, $\sigma_{i}$, is said to be injective modulo $\Delta_{i}$ if for any two variables $x, y$ in the domain of $\sigma_{i}$, we have that $x \sigma_{i}=\Delta_{i} y \sigma_{i}$ if and only if $x=y$.

\section{Definition 7. (Theory Preserving)}

A substitution $\sigma_{i}$, solving an $i$-pure problem $\Gamma_{i}$, is said to be theory preserving if for any variable $x$ of index $i$ in the domain of $\sigma_{i}, x \sigma_{i}$ is not a variable of index $j \neq i$.

With respect to the combination algorithm this definition basically states that a substitution $\sigma_{i}$, which solves an $i$-pure problem, $\Gamma_{i}$, produced by Algorithm AsymComb (cf. Figure 1), is theory-preserving if for all $x \in \operatorname{Dom}\left(\sigma_{i}\right)$, $x \sigma_{i} \neq c$ where $c$ is a free constant. This is due to the fact that for the pure sub-problems produced by the combination algorithm the only free constants 
will be those corresponding to shared variables of a different index. Note, the definition of theory-preserving does not restrict $\sigma_{i}$ from sending an $i$-variable $x$ to a non-variable $i$-term whose leafs are $j$-variables. Thus, if $x \sigma_{i}=t$ and $t$ is an $i$-term, then $t$ may contain $j$-variables. In addition, since the Algorithm Asym$C o m b$ assigns the variable indexes, it can always check the substitutions returned by the algorithms for the pure theories to ensure that they are injective and theory-preserving.

Definition 8. Let $\sigma_{1}$ and $\sigma_{2}$ be unifiers with linear constant restriction for $\Gamma_{5,1}$ and $\Gamma_{5,2}$ such that $\Gamma_{5, i}$ is the set of $i$-pure equations from $\Gamma_{4}$ and $<$ is the corresponding linear ordering. The combined solution $\sigma_{1} \odot \sigma_{2}$ is defined by induction on $<$ :

Let $x$ be the least variable with respect to the ordering $<$ from step 4 and let $i$ be its index. Since the solution $\sigma_{i}$ of $\Gamma_{5, i}$ satisfies the constant restriction induced by $<, x \sigma_{i}$ does not contain any variables of index $j \neq i$. We define $x\left(\sigma_{1} \odot \sigma_{2}\right)$ to be $x \sigma_{i}$.

Let $x$ be an arbitrary variable of index $i$ and let $y_{1}, \ldots, y_{m}$ be the variables of index $j \neq i$ occurring in $x \sigma_{i}$. Again, due to the constant restriction, the variables $y_{1}, \ldots, y_{m}$ have to be smaller than $x$. This implies that $y_{1}\left(\sigma_{1} \odot \sigma_{2}\right), \ldots, y_{m}\left(\sigma_{1} \odot\right.$ $\left.\sigma_{2}\right)$ are already defined. The term $x\left(\sigma_{1} \odot \sigma_{2}\right)$ is obtained from $x \sigma_{i}$ by replacing $y_{k}$ by $y_{k}\left(\sigma_{1} \odot \sigma_{2}\right)$, and we define $x\left(\sigma_{1} \odot \sigma_{2}\right)$ to be $x \sigma_{i}\left(\sigma_{1} \odot \sigma_{2}\right)$.

Lemma 3. (Baader-Schulz [2])

The combined unifier $\sigma_{1} \odot \sigma_{2}$ from Definition 8 is a unifier of $\Gamma_{4}$.

Example 2. Let $\Delta_{1}=R_{1} \cup E_{1}$, where $R_{1}=\{e(x, d(x, y)) \rightarrow y, d(x, e(x, y)) \rightarrow y\}$ and $E_{1}=\emptyset$. Let $\Delta_{2}=R_{2} \cup E_{2}$, where $R_{2}=\{x \oplus 0 \rightarrow x, x \oplus x \rightarrow 0, x \oplus x \oplus y \rightarrow y\}$ and $\left.E_{2}=\{x \oplus y=y \oplus x,(x \oplus y) \oplus z=x \oplus(y \oplus z)\}\right\}$. Let $\Delta=\Delta_{1} \cup \Delta_{2}$.

Consider the initial problem $\Gamma_{0}$ consisting of the following: $\left\{x_{0} \oplus x_{1} \oplus x_{2}=\downarrow\right.$ $\left.x_{3} \oplus x_{4}, e\left(x_{1}, d\left(0, x_{5}\right)\right)=\downarrow x_{2} \oplus x_{0}, e\left(x_{1}, d\left(x_{0}, e\left(x_{2}, x_{6}\right)\right)\right)=\downarrow e\left(x_{7}, x_{5}\right)\right\}$

Let us now examine the action of Algorithm AsymComb (cf. Figure 1) on $\Gamma_{0}$ and how it would find a particular asymmetric solution. The first 2 steps produce the set of pure equations $\Gamma_{2}:\left\{x_{0} \oplus x_{1} \oplus x_{2}=^{\downarrow} x_{3} \oplus x_{4}, e\left(x_{1}, d\left(z_{0}, x_{5}\right)\right)={ }^{\downarrow} z_{1}, 0=\downarrow\right.$ $\left.z_{0}, z_{1}=\downarrow x_{2} \oplus x_{0}, e\left(x_{1}, d\left(x_{0}, e\left(x_{2}, x_{6}\right)\right)\right)=\downarrow e\left(x_{7}, x_{5}\right)\right\}$.

The next step considers the set of variable partitions, one of which is the following partition $\left\{\left\{x_{0}, x_{3}\right\},\left\{x_{2}, x_{4}\right\},\left\{x_{5}, z_{1}\right\},\left\{x_{1}, z_{0}, x_{7}\right\},\left\{x_{6}\right\}\right\}$ Choosing a representative for each set and doing the replacement the Algorithm would produce the following $\Gamma_{3}$ from that partition: $\left\{x_{0} \oplus x_{1} \oplus x_{2}=^{\downarrow} x_{0} \oplus x_{2}, e\left(x_{1}, d\left(x_{1}, x_{5}\right)\right)=\downarrow\right.$ $\left.x_{5}, 0=\downarrow x_{1}, x_{5}=^{\downarrow} x_{2} \oplus x_{0}, e\left(x_{1}, d\left(x_{0}, e\left(x_{2}, x_{6}\right)\right)\right)={ }^{\downarrow} e\left(x_{1}, x_{5}\right)\right\}$.

The next step considers the possible pairs of variable orderings and theory indexes. One pair that would be produced is the following: $x_{6}>x_{5}>x_{2}>x_{1}>$ $x_{0}$, index-1 $=\left\{x_{0}, x_{1}, x_{2}, x_{5}\right\}$ and index- $2=\left\{x_{6}\right\}$.

Next $\Gamma_{4}$ is produced from that pair and split into pure sets to produce $\Gamma_{5,1}$ and $\Gamma_{5,2}$. Let us denote a variable, $y$, being treated as a constant as $\mathbf{y}$. Then, $\Gamma_{5,1}$ is the following set of equations: $\left\{x_{0} \oplus x_{1} \oplus x_{2}=\downarrow x_{0} \oplus x_{2}, 0=\downarrow\right.$ $\left.x_{1}, x_{5}=\downarrow x_{2} \oplus x_{0}\right\}$ and $\Gamma_{5,2}$ is the following set of equations: $\left\{e\left(\mathbf{x}_{1}, d\left(\mathbf{x}_{1}, \mathbf{x}_{5}\right)\right)=\downarrow\right.$ $\left.\mathbf{x}_{5}, e\left(\mathbf{x}_{1}, d\left(\mathbf{x}_{0}, e\left(\mathbf{x}_{2}, x_{6}\right)\right)\right)={ }^{\downarrow} e\left(\mathbf{x}_{1}, \mathbf{x}_{5}\right)\right\}$ 
Next $\Gamma_{5,1}$ is solved with $A_{1}$ and $\Gamma_{5,2}$ with $A_{2}$, where the linear constant restriction is obtained via the linear ordering and theory index. The last step is to combine each pair of substitutions $\left(\sigma_{1}, \sigma_{2}\right)$ into a substitution $\sigma$, where $\sigma_{i}$ is an injective and theory-preserving asymmetric with LCR solution to $\Gamma_{5, i}$ returned by $A_{i}$. One such pair is $\sigma_{1}=\left\{x_{1} \mapsto 0, x_{5} \mapsto x_{2} \oplus x_{0}\right\}$ and $\sigma_{2}=$ $\left\{x_{6} \mapsto d\left(\mathbf{x}_{2}, e\left(\mathbf{x}_{0}, \mathbf{x}_{5}\right)\right)\right\}$. Applying Definition 8 we get the following solution, $\left\{x_{1} \mapsto 0, x_{3} \mapsto x_{0}, x_{4} \mapsto x_{2}, x_{5} \mapsto x_{2} \oplus x_{0}, x_{6} \mapsto d\left(x_{2}, e\left(x_{0}, x_{2} \oplus x_{0}\right)\right), x_{7} \mapsto 0\right\}$, which is an asymmetric solution to $\Gamma_{0}$ (existential variables $z_{0}, z_{1}$ are removed).

Before presenting the proof details lets us briefly point out the main differences between Algorithm AsymComb (cf. Figure 1) and the algorithm of [2]. While the general framework of the two algorithms is similar there are several key differences. First, we do not consider general theories. Due to the restrictions inherited from the definition of asymmetric unification we must consider theories with specific structure, namely the decomposition. This requires new results for showing the correctness of the algorithm and new results for showing that the required properties for asymmetric unification are maintained. Second, we must identify the specific unifiers which satisfy the asymmetry. We accomplish this by identifying two key properties, theory preservation (Definition. 7) and injectivity (Definition. 6).

\subsection{Correctness}

We show in this section that the Algorithm AsymComb (cf. Figure 1) is both sound and complete for the decision problem. In addition, we show that the algorithm produces a complete set of asymmetric unifiers.

Lemma 4. Assume that $\sigma_{1}$ and $\sigma_{2}$ are pure, injective, theory-preserving and $R_{i}, E_{i}$-normalized unifiers modulo respectively $\Delta_{1}=R_{1} \uplus E_{1}$ and $\Delta_{2}=R_{2} \uplus E_{2}$ and they satisfy the same linear constant restriction. Then, the substitution $\sigma=$ $\sigma_{1} \odot \sigma_{2}$ satisfies the following properties: (1) $\sigma$ is an injective substitution modulo $\Delta_{1} \cup \Delta_{2}$. (2) $\sigma$ is $\mathcal{R}, \mathcal{E}$-normalized.

Proof. We proceed by induction on the linear ordering.

Base case: Let $v$ be the smallest variable, say of index $i$. Then, $\sigma$ is clearly injective and $\mathcal{R}, \mathcal{E}$-normalized for variables smaller or equal to $v$, since $v \sigma=v \sigma_{i}$ is $R_{i}, E_{i}$-normalized, and so also $\mathcal{R}, \mathcal{E}$-normalized.

Inductive case: Assume the the properties holds for variables smaller than a variable $y$ of index $i$. To show that (1) holds, assume by contradiction that there exists a variable $x$ strictly smaller than $y$ such that $x \sigma=\Delta_{1} \cup \Delta_{2} y \sigma$. Since $\sigma$ is $\mathcal{R}, \mathcal{E}$-normalized for variables smaller than $y$, we have that $x \sigma={ }_{\Delta_{1}} \cup \Delta_{2}$ $y \sigma$ implies $x \sigma^{\pi_{i}}=\Delta_{i} y \sigma^{\pi_{i}}$. Since $\sigma$ is injective for variables smaller than $y$, there exists a renaming $\rho$ such that $x \sigma^{\pi_{i}} \rho=x \sigma_{i}$ and $y \sigma^{\pi_{i}} \rho=y \sigma_{i}$. Therefore $x \sigma_{i}=\Delta_{i} y \sigma_{i}$, which is a contradiction. Consider now the property (2): if $y \sigma$ is $\mathcal{R}, \mathcal{E}$-reducible, then $(y \sigma)^{\pi_{i}}$ is $R_{i}, E_{i}$-reducible, which means that $y \sigma_{i} \rho$ and $y \sigma_{i}$ are $R_{i}, E_{i}$-reducible too, which contradicts the assumption that $\sigma_{i}$ is an $R_{i}$, $E_{i}$-normalized substitution. 
Lemma 5. Let $\Gamma_{0}$ be a solvable asymmetric $\Delta$-unification problem, where $\Delta=$ $\Delta_{1} \cup \Delta_{2}$. Assume there exists a pair $\left(\Gamma_{5,1}, \Gamma_{5,2}\right)$ produced by the Algorithm AsymComb (cf. Figure 1) on $\Gamma_{0}$ and a pair $\left(\sigma_{1}, \sigma_{2}\right)$ such that $\sigma_{i} \in C S A U_{\Delta_{i}}\left(\Gamma_{5, i}\right)$ for $i=1,2$.

Then, there exists pairs $\left(\Gamma_{5,1}^{\prime}, \Gamma_{5,2}^{\prime}\right)$ produced by the Algorithm AsymComb on $\Gamma_{0}$ and a pair $\left(\phi_{1}, \phi_{2}\right)$ such that $\phi_{i}$ is injective and theory-preserving, $\phi_{i} \in$ $C S A U_{\Delta_{i}}\left(\Gamma_{5, i}^{\prime}\right)$ for $i=1,2$, and $\phi_{1} \odot \phi_{2} \leq_{\Delta}^{\operatorname{Var}\left(\Gamma_{0}\right)} \sigma_{1} \odot \sigma_{2}$.

Proof. Construct $\left(\Gamma_{5,1}^{\prime}, \Gamma_{5,2}^{\prime}\right)$ and $\left(\sigma_{1}^{\prime}, \sigma_{2}^{\prime}\right)$ : Let $\Gamma_{4}$ be the conjunction of $\Gamma_{5,1}$ and $\Gamma_{5,2}$. Then there exists a linear ordering, $<$, and a theory index, ind. From $\Gamma_{4}$ we can construct a new $\Gamma_{4}^{\prime}$ as follows: If there exists $x, y$ in the domain of $\sigma_{i}$ such that $x \sigma_{i}=\Delta_{i} y \sigma_{i}$ we add $x=y$ to the variable identification. If there exists variables $x, y$ such that $x$ is an index $i$ variable, $y$ is an index $j$ variable and $x \sigma_{i}=y$, we replace all $x$ with $y$ in the variable identification. $i$ ind and $<$ remain the same. The result of these steps is a new $\Gamma_{4}^{\prime}$, which also gives us a new pair $\left(\Gamma_{5,1}^{\prime}, \Gamma_{5,2}^{\prime}\right)$. We can now define the new pair of substitutions $\left(\sigma_{1}^{\prime}, \sigma_{2}^{\prime}\right)$ as follows: Let $\operatorname{Dom}\left(\sigma_{i}^{\prime}\right)=\operatorname{Var}\left(\Gamma_{5, i}^{\prime}\right) . \forall x \in \operatorname{Dom}\left(\sigma_{i}^{\prime}\right), x \sigma_{i}^{\prime}=x \sigma_{i}$ and is the identity on all other variables.

Show that $\sigma_{1}^{\prime}$ and $\sigma_{2}^{\prime}$ are theory-preserving and injective unifiers of $\Gamma_{5,1}^{\prime}$ and $\Gamma_{5,2}^{\prime}$ : This follows from the construction of $\Gamma_{4}^{\prime}$, where variables violating the definitions have been removed.

Show that $\forall x \in \operatorname{Var}\left(\Gamma_{0}\right) x \sigma={ }_{\Delta} x \sigma^{\prime}$ : First, by the definition of $\sigma^{\prime}$ for all $x \in$ $\operatorname{Dom}\left(\sigma^{\prime}\right), x \sigma^{\prime}=x \sigma$. Therefore, we need only consider the variables removed by the variable identification step. From Definition 8 , for any variable $x$ in the initial system replaced by a variable $y$ during the identification step, $x \sigma:=y \sigma$. Since any identifications occuring in the definition of $\Gamma_{4}$ must also occur in $\Gamma_{4}^{\prime}, x \sigma^{\prime}:=y \sigma^{\prime}=y \sigma=x \sigma$. Now consider the variable identifications added to construct $\Gamma_{4}^{\prime}$ but not existing in $\Gamma_{4}$. If $x=y$ is added because $x \sigma_{i}=y \sigma_{i}$, without loss of generality assume $x$ is replaced by $y$, then $x \sigma^{\prime}:=y \sigma^{\prime}=y \sigma={ }_{\Delta} x \sigma$. Lastly if $x$ is replaced by $y$ because $x \sigma_{i}=y$, then $x \sigma^{\prime}:=y \sigma=x \sigma$.

To complete the proof, there exists $\phi_{i} \in C S A U_{\Delta}\left(\Gamma_{5, i}^{\prime}\right)$ such that $\phi_{i} \leq_{\Delta}^{\operatorname{Var}\left(\Gamma_{0}\right)}$ $\sigma_{i}^{\prime}$ for $i=1,2$. By the definition of $\odot$, we have that $\phi_{1} \odot \phi_{2} \leq_{\Delta}^{\operatorname{Var}\left(\Gamma_{0}\right)} \sigma_{1}^{\prime} \odot \sigma_{2}^{\prime}=\sigma^{\prime}$, and $\sigma^{\prime}={ }_{\Delta}^{\operatorname{Var}\left(\Gamma_{0}\right)} \sigma$. Therefore, $\phi_{1} \odot \phi_{2} \leq_{\Delta}^{\operatorname{Var}\left(\Gamma_{0}\right)} \sigma_{1} \odot \sigma_{2}=\sigma$.

Lemma 6. For each asymmetric unifier of a problem $\Gamma_{0}$, there exists a pair $\left(\Gamma_{5,1}, \Gamma_{5,2}\right)$ computed by the Algorithm AsymComb (cf. Figure 1), where for each $\Gamma_{5, i}$ there exist a substitution $\tau_{i}$ which asymmetrically solves $\Gamma_{5, i}$.

Proof. The construction given in 2] can be used here with modifications to account for asymmetry and the projection $\pi$ (Equation 1), see [9].

Lemma 7. Let $\Gamma_{0}$ be an asymmetric $\Delta$-unification problem. For each asymmetric unifier $\tau$ of $\Gamma_{0}$ there exists a pair $\left(\Gamma_{5,1}, \Gamma_{5,2}\right)$ in the output set of the Algorithm AsymComb (cf. Figure 1), and a pair of substitutions $\left(\sigma_{1}, \sigma_{2}\right)$ with each $\sigma_{i} \in C S A U_{\Delta_{i}}\left(\Gamma_{5, i}\right)$. Such that $\sigma=\sigma_{1} \odot \sigma_{2}$ is an injective asymmetric solution to $\Gamma_{0}$ with $\sigma \leq_{\Delta}^{\operatorname{Var}\left(\Gamma_{0}\right)} \tau$. 
Proof. From Lemma 6 we have that given $\tau$ there exists a pair $\left(\Gamma_{5,1}^{1}, \Gamma_{5,2}^{1}\right)$ and substitutions $\left(\tau_{1}^{1}, \tau_{2}^{1}\right)$ such that $\tau_{i}^{1}$ asymmetrically solves $\Gamma_{5, i}^{1}$. Now, if $\tau_{i}^{1}$ is an asymmetric solution to $\Gamma_{5, i}^{1}$, there exists a substitution $\tau_{i}^{2}$ produced by the algorithm $A_{i}$ such that $\tau_{i}^{2} \in C S A U_{\Delta_{i}}\left(\Gamma_{5, i}^{1}\right)$ and $\tau_{i}^{2} \leq_{\Delta_{i}}^{\operatorname{Var}\left(\Gamma_{5, i}\right)} \tau_{i}^{1}$. Furthermore, as in Lemma 5 , by the definition of $\odot$, we have that $\tau^{2}=\tau_{1}^{2} \odot \tau_{2}^{2} \leq_{\Delta}^{\operatorname{Var}\left(\Gamma_{0}\right)} \tau_{1}^{1} \odot \tau_{2}^{1}=\tau$

From Lemma 5, there exists a pair $\left(\Gamma_{5,1}^{2}, \Gamma_{5,2}^{2}\right)$ produced by AsymComb and a pair $\left(\sigma_{1}, \sigma_{2}\right)$ such that $\sigma_{i}$ is injective and theory-preserving, $\sigma_{i} \in C S A U_{\Delta_{i}}\left(\Gamma_{5, i}^{2}\right)$ and $\sigma=\sigma_{1} \odot \sigma_{2} \leq_{\Delta}^{\operatorname{Var}\left(\Gamma_{0}\right)} \tau^{2}$. By Lemma 4, $\sigma$ is an injective asymmetric solution to $\Gamma_{4}$. Finally, $\sigma \leq_{\Delta}^{\operatorname{Var}\left(\Gamma_{0}\right)} \tau^{2}$ and $\tau^{2} \leq_{\Delta}^{\operatorname{Var}\left(\Gamma_{0}\right)} \tau$, and so $\sigma \leq_{\Delta}^{\operatorname{Var}\left(\Gamma_{0}\right)} \tau$.

We can now show the the Algorithm AsymComb (cf. Figure1) is correct, i.e., both sound and complete.

Theorem 5. Let $\Gamma_{0}$ be a combined asymmetric unification problem. $\Gamma_{0}$ is asymmetrically unifiable if and only if the Algorithm AsymComb (cf. Figure 1) returns a combined substitution.

Proof. From Lemma 3 the substitutions returned are unifiers. From Lemma 4 the substitutions are asymmetric. Completeness follows from Lemma 7.

Now we can consider the complete set of unifiers.

Theorem 6. Let $\Gamma_{0}$ be an asymmetric $\Delta$-unification problem. Then, for every $\tau \in C S A U\left(\Gamma_{0}\right)$, Algorithm AsymComb (cf. Figure 1) produces an injective substitution $\sigma$ such that $\sigma \leq_{\Delta}^{\operatorname{Var}\left(\Gamma_{0}\right)} \tau$.

Proof. For any problem, $\Gamma_{0}$, the Algorithm AsymComb will try every combination of variable identification, theory index and linear ordering, i.e. every possible pair of sub-problems $\left(\Gamma_{5,1}, \Gamma_{5,2}\right)$. Furthermore, the Algorithm AsymComb will combine every pair, $\left(\sigma_{1}, \sigma_{2}\right)$, of injective and theory preserving solutions such that $\sigma_{i} \in C S A U_{\Delta_{i}}\left(\Gamma_{5, i}\right), i \in\{1,2\}$. Thus, the result follows from Lemma 7

\subsection{Obtaining Linear Constant Restriction Algorithms}

If one has a general asymmetric unification algorithm an algorithm that respects an LCR can be obtained. The construction is similar to the one given in [2] Given $\Gamma$, an asymmetric unification problem with a linear constant restriction, we construct a general unification problem $\Gamma^{\prime}$ such that $\Gamma$ is solvable iff $\Gamma^{\prime}$ is solvable. Let $<$ denote the linear ordering. Let $\mathcal{X}$ denote the variables of $\Gamma$ and let $C$ denote the set of all free constants in $\Gamma$. Now, we construct $\Gamma^{\prime}$ as follows: The free constants in $\Gamma$ are treated as variables in $\Gamma^{\prime}$. For each free constant $c$ of $\Gamma$ we add a new free function symbol $f_{c}$ which has arity $\left|V_{c}\right|$. Recall that $V_{c}=$ $\{x \in \mathcal{X} \mid x<c\} . \Gamma^{\prime}=\Gamma \cup\left\{c=\downarrow f_{c}\left(x_{1}, \ldots, x_{n}\right) \mid c \in C\right.$ and $\left.V_{c}=\left\{x_{1}, \ldots, x_{n}\right\}\right\}$

Theorem 7. The Asymmetric E-unification problem with linear constant restriction, $\Gamma$, is solvable iff the general Asymmetric E-unification problem $\Gamma^{\prime}$ is solvable.

Proof. The same proof used in [2] can here with only a modification for asymmetric equations. A full proof is given in [9]. 


\section{Conclusions}

With respect to efficiency, the combination algorithm provides a significant first step to more efficient methods since, unlike a narrowing approach, we can now combine efficient special purpose asymmetric unification algorithms. In addition, it should be possible to improve the efficiency of the current algorithm. We are currently studying the question of improving the efficiency.

Briefly, the only theories that are currently known to have asymmetric unification algorithms are those with the finite variant property [6], in which case a general algorithm known as folding variant narrowing [12] applies. This is a sizable class, including many, but not all, theories of interest to cryptographic protocol analysis (see [6]). In many cases known characterizations of theories with the finite variant property [12, [5] depend on conditions on $E$ and $R$ that can be checked without further reference to $\Sigma$, and so for these cases the finite variant property still holds after the addition of uninterpreted function symbols. Thus general asymmetric unification algorithms exist. Moreover, the earlier mentioned special-purpose algorithms for exclusive-or and free Abelian groups [14, [8] are also general asymmetric algorithms. In [14] and 8] a strategy is presented for converting symmetric unification algorithms to asymmetric ones. This opens up an avenue for the development of special-purpose general asymmetric unification algorithms for theories with and without the finite variant property as well, to which our results would also apply.

There exists an interesting connection between Asymmetric unification and Disunification. Consider a disunification problem $s \neq t$ in the theory $\Delta=E \cup R$ over signature $\Sigma$. We can simulate this problem using asymmetric unification. First, let $f$ and $g$ be new function symbols added to $\Sigma$. Let $f(x, x) \longrightarrow g(x)$ be a new rule added to $R$. Now $s \neq t$ can be simulated by $\{s=\downarrow u, t=\downarrow v, w=\downarrow$ $f(u, v)\}$. Although there is some connection between the two problems they may still be independent and resolving this is an interesting open problem.

\section{References}

1. Baader, F., Nipkow, T.: Term rewriting and all that. Cambridge University Press, New York (1998)

2. Baader, F., Schulz, K.U.: Unification in the Union of Disjoint Equational Theories: Combining Decision Procedures. Journal of Symbolic Computation 21(2), 211-243 (1996)

3. Baader, F., Snyder, W.: Unification Theory. In: Robinson, J.A., Voronkov, A. (eds.) Handbook of Automated Reasoning, pp. 445-532. Elsevier and MIT Press (2001)

4. Blanchet, B.: An Efficient Cryptographic Protocol Verifier Based on Prolog Rules. In: Proceedings of the 14th IEEE Workshop on Computer Security Foundations, CSFW 2001, pp. 82-96. IEEE Computer Society (2001)

5. Bouchard, C., Gero, K.A., Lynch, C., Narendran, P.: On Forward Closure and the Finite Variant Property. In: Fontaine, P., Ringeissen, C., Schmidt, R.A. (eds.) FroCoS 2013. LNCS, vol. 8152, pp. 327-342. Springer, Heidelberg (2013)

6. Comon-Lundh, H., Delaune, S.: The Finite Variant Property: How to Get Rid of Some Algebraic Properties. In: Giesl, J. (ed.) RTA 2005. LNCS, vol. 3467, pp. 294-307. Springer, Heidelberg (2005) 
7. Erbatur, S., Escobar, S., Kapur, D., Liu, Z., Lynch, C., Meadows, C., Meseguer, J., Narendran, P., Santiago, S., Sasse, R.: Effective Symbolic Protocol Analysis via Equational Irreducibility Conditions. In: Foresti, S., Yung, M., Martinelli, F. (eds.) ESORICS 2012. LNCS, vol. 7459, pp. 73-90. Springer, Heidelberg (2012)

8. Erbatur, S., Escobar, S., Kapur, D., Liu, Z., Lynch, C.A., Meadows, C., Meseguer, J., Narendran, P., Santiago, S., Sasse, R.: Asymmetric Unification: A New Unification Paradigm for Cryptographic Protocol Analysis. In: Bonacina, M.P. (ed.) CADE 2013. LNCS, vol. 7898, pp. 231-248. Springer, Heidelberg (2013)

9. Erbatur, S., Kapur, D., Marshall, A.M., Meadows, C., Narendran, P., Ringeissen, C.: On Asymmetric Unification and the Combination Problem in Disjoint Theories. INRIA Research Report (2014), http://hal.inria.fr/

10. Escobar, S., Meadows, C., Meseguer, J.: Maude-NPA: Cryptographic Protocol Analysis Modulo Equational Properties. In: Aldini, A., Barthe, G., Gorrieri, R. (eds.) FOSAD 2007/2008/2009. LNCS, vol. 5705, pp. 1-50. Springer, Heidelberg (2009)

11. Escobar, S., Meseguer, J., Sasse, R.: Variant Narrowing and Equational Unification. Electronic Notes Theor. Comput. Science 238(3), 103-119 (2009)

12. Escobar, S., Sasse, R., Meseguer, J.: Folding Variant Narrowing and Optimal Variant Termination. J. Log. Algebr. Program. 81(7-8), 898-928 (2012)

13. Jouannaud, J.-P., Toyama, Y.: Modular Church-Rosser Modulo: The Complete Picture. Int. J. Software and Informatics 2(1), 61-75 (2008)

14. Liu, Z.: Dealing Efficiently with Exclusive OR, Abelian Groups and Homomorphism in Cryptographic Protocol Analysis. PhD thesis, Clarkson University (2012)

15. Meier, S., Schmidt, B., Cremers, C., Basin, D.: The TAMARIN Prover for the Symbolic Analysis of Security Protocols. In: Sharygina, N., Veith, H. (eds.) CAV 2013. LNCS, vol. 8044, pp. 696-701. Springer, Heidelberg (2013)

16. Mödersheim, S.: Models and methods for the automated analysis of security protocols. PhD thesis, ETH Zurich (2007)

17. Rusinowitch, M.: On Termination of the Direct sum of Term-Rewriting Systems. Information Processing Letters 26, 65-70 (1987) 